\title{
Meta-analysis of economic evaluation studies: Data Harmonisation and Methodological issues
}

Bhavani Shankara Bagepally

ICMR-National Institute of Epidemiology

\section{Usa Chaikledkaew}

Mahidol University Health Technology Assessment (MUHTA), Mahidol University

\section{Nathorn Chaiyakunapruk}

University of Utah

John Attia

University of Newcastle

Ammarin Thakkinstian ( $\square$ ammarin.tha@mahidol.edu )

Mahidol University Health Technology Assessment (MUHTA), Mahidol University

\section{Research Article}

Keywords: Economic evaluation, CUA, Cost-effectiveness

Posted Date: September 30th, 2021

DOI: https://doi.org/10.21203/rs.3.rs-93779/v2

License: (c) (1) This work is licensed under a Creative Commons Attribution 4.0 International License.

Read Full License

Version of Record: A version of this preprint was published at BMC Health Services Research on February 15th, 2022. See the published version at https://doi.org/10.1186/s12913-022-07595-1. 
Title: Meta-analysis of economic evaluation studies: Data Harmonisation and Methodological issues

3 Short title: Meta-analysis of economic evaluation studies

4 Authors:

5

- Bhavani Shankara Bagepally ${ }^{1,2}, \mathrm{MBBS}, \mathrm{MSc}, \mathrm{PhD}$

- Usa Chaikledkaew ${ }^{1,3}, \mathrm{PhD}$

- Nathorn Chaiyakunapruk ${ }^{4}$, PharmD, PhD

- John Attia ${ }^{5}, \mathrm{MD}, \mathrm{PhD}$

- Ammarin Thakkinstian ${ }^{1,6^{*}}, \mathrm{PhD}$

\section{Author Affiliation:}

1. Mahidol University Health Technology Assessment (MUHTA) Graduate Program, Mahidol University, Bangkok, Thailand

2. ICMR-National Institute of Epidemiology, Chennai, India

3. Social Administrative Pharmacy Division, Department of Pharmacy, Faculty of Pharmacy, Mahidol University, Bangkok, Thailand

4. Department of Pharmacotherapy, College of Pharmacy, University of Utah, Utah, USA

5. Centre for Clinical Epidemiology \& Biostatistics, Hunter Medical Research Institute, School of Medicine and Public Health, University of Newcastle, New Lambton, NSW, Australia

6. Department of Clinical Epidemiology and Biostatistics, Faculty of Medicine Ramathibodi Hospital, Mahidol University, Bangkok, Thailand

\section{Authors Contribution:}

- BSB: Conceptualization; Data curation, Formal analysis, Writing - original draft

- UC: Supervision, Writing - review \& editing

- NC: Writing - review \& editing

- JA: Writing - review \& editing

- AT: Conceptualization, Methodology, Supervision, Writing - review \& editing

Ethics Approval: Since the work is methods work on Systematic review and meta-analysis of published literature which is exempted from formal ethical review as per national ICMR Guidelines.

\section{*Corresponding Author:}

Ammarin Thakkinstian,

Department of Clinical Epidemiology and Biostatistics,

Faculty of Medicine Ramathibodi Hospital,

Rama VI Road, Bangkok, 10400 Thailand

Email: ammarin.tha@mahidol.edu

Word counts: Abstract $=163$, Text $=3211$, Number of Tables: 4, Number of Figures: 1 , Key words: Economic evaluation, CUA, Cost-effectiveness. 


\section{Abstract:}

2 Background: In the context of ever-growing health expenditure and limited resources, economic evaluations aid in making evidence-informed policy decisions. Cost utility analyses

4 (CUA) are often used in this context, but limitations include pairwise contrasts, missing 5 contrasts, and different sources or quality of data.

6 Results: Synthesis of CUA data from multiple studies is therefore desirable to assist policy

7 makers, but there are many challenging methodological issues including: inconsistent reporting

8 of results using different economic parameters, and multiple sources of heterogeneity 9 including: setting, time horizon, perspective, modelling approaches and assumptions, currency, 10 willingness to pay (WTP) threshold, level of country income, and input parameters. In this paper, we provide a step by step description of the methods for data harmonisation and synthesis of aggregated data from CUA studies, as well as a framework for handling heterogeneity; we demonstrate these methods using the example of agents for type 2 diabetes.

Conclusion: These meta-analytic methods for the synthesis of economic evidence synthesis should be useful for policy makers. 


\section{Background:}

2 In the context of ever-growing health expenditure and limited resources, identifying healthcare services that yield the highest benefit at the lowest cost is a priority. Economic evaluation studies (EES) provide a framework to systematize both clinical and economic outcomes[1] Cost-utility analysis (CUA) is commonly applied to compare clinical and economic outcomes by estimating an incremental cost-effectiveness ratio (ICER). The costs are usually measured in a specific country currency, while the health benefit is usually measured as a quality adjusted life year (QALY), i.e., the product of years lived and health utility score ranging from 0 (death) to 1 (perfect health), or disability adjusted life years (DALY)[2, 3]. The ICER is calculated by $\left(\right.$ Cost $_{\text {intervention }}-$ Cost $\left._{\text {comparator }}\right) /\left(\mathrm{QALY}_{\text {intervention }}-\mathrm{QALY}_{\text {comparator }}\right)$, if the ICER is under the willingness to pay (WTP) threshold (measured in monetary cost per QALY gained), the health intervention is considered to be cost-effective.

However, many methodological issues in the data synthesis of EESs are more challenging than clinical studies because there are many sources of heterogeneity including: study characteristics (e.g., setting, WTP, country, country income), methodology (time horizon, perspective, data source, model type, input parameters, and assumptions). This is perhaps why most previous systematic reviews of EESs have performed only descriptive analyses and reported only qualitative findings without applying a meta-analysis (MA) to estimate pooled effect measures. Although Crespo et al [4] have described a MA for pooling EES (known as the COMparative Efficiency Research, COMER), it has yet been widely adopted as such MA for clinical outcomes. This might be due to the fact either EESs are too heterogeneous to pool or they chose the lesser known parameter "incremental net benefit" (INB) as the effect measure rather than the more commonly used ICER. However, we believe the choice for pooling INB was justified due to the limitations of the ICER [5]. For instance, a negative ICER may indicate a lower cost compared with higher effectiveness or higher cost along with lower effectiveness of 
1 interventions, thus introducing ambiguity in interpretation $[4,6]$. In contrast, positive and

2 negative INBs directly indicate cost-effectiveness and non-cost-effectiveness of interventions,

3 respectively, which is the information required by policy makers [7, 8]. Furthermore, the

4 COMER method mainly focused on the statistical methods for pooling, but did not suggest a

5 step-by-step process for data harmonisation and processing to aid the synthesis of economic

6 studies.

7 Hence, in this tutorial paper, we provide step by step of the methods for data harmonisation and synthesis of EESs. Data for cost-effectiveness of diabetic drug controls are used as a demonstration.

\section{Methods}

Methods are similar to other systematic reviews and MAs [9, 10], and should follow the Preferred Reporting Items for Systematic Reviews and Meta-Analyses (PRISMA) [11] guidelines. This methodological study was a part of previous MAs, in which some additional specific issues apply to EESs are as follows[12-15].

\section{Step 1: Data extraction}

All relevant data for comparative EESs (e.g., CUA) should be extracted as follows utilising the PICOS (Population Intervention Comparator Outcome and Study type) framework:

- General characteristics of EESs including study setting/country, study design (e.g., CUA with model-based, primary CUA alongside RCT/cohort), study perspective, time horizon, discount rate for cost and utility, type of EESs (e.g., CUA or cost-effectiveness analysis (CEA)), willingness to pay (WTP; country specific or gross domestic products (GDP)-base) or country-level cost-effectiveness threshold[16] where appropriate if WTP was not available, and type of economic models.

- Characteristics of patients (P) including indication for treatment, sample size, type of 
patients (e.g., children/adult, general/specific disease, etc.), mean age, percent male/female, mean body mass index (BMI), etc.

- Type of interventions and comparators (I \& C) (with duration of treatment/dosage/day, etc.)

- Data needed to estimate INB and its variance (O); this includes currency and year, source of cost data (actual data cost collected from patients, central hospital/country costs, etc.) type of cost (e.g., direct/non-direct medical cost, indirect medical cost), and effectiveness outcomes (e.g., life year, QALY, ICER, etc.).

Specific data required for pooling include costs or incremental cost $(\Delta \mathrm{C})$, and incremental effectiveness $(\Delta \mathrm{E})$ along with their standard deviation (SD), standard error (SE), or $95 \%$ confidence interval (CI). Some studies may report ICER along with one-way and/or probabilistic sensitivity analysis (PSA). To calculate the INB and its variance, mean and variance of the costs and effectiveness of interventions and comparators along with WTP thresholds are required. In the model-based CUA, studies usually report point estimates of deterministic and/or probabilistic costs and outcomes. We suggest using primarily the measures of central tendency and dispersion measures from PSA results for pooling, as it could be a better representative of real-life situation considering the distribution of all input variables, and conducting sensitivity analyses using point estimates from the deterministic analysis to see robustness of results. The WTP threshold was initiated by the Commission on Macroeconomics and Health in 2002 by the World Health Organization CHOosing Interventions that are CostEffective (WHO CHOICE). The WTP threshold in each country usually refers from the standard country guideline based on a fixed value or per capita GDP with returns on investments in health to define whether a health intervention would be (very) cost-effective. $[17,18]$. We suggest using the same WTP threshold in monetary units used in the study with further adjustment as per currency conversions as mentioned below. 


\section{Step 2: Data harmonisation}

\section{Currency conversions}

3 We need to standardize money units usually reported in different currencies (i.e., US $\$, €, £$,

$4 ¥)$ and years by converting to purchasing power parity (PPP) adjusted to US\$ for the latest year

5 of analysis. For example, if a study reported cost, ICER, and thresholds in Euros for 2012 and

6 we plan to pool for the current year (e.g., 2020), this currency is firstly converted to 2020 Euros

7 using the historical consumer price index (CPI) of that country. Then, the Euro 2020 value is

8 converted to PPP adjusted US\$ rate using conversion rates from the International Monetary

9 Fund [19]. In addition, GDP-based WTP threshold (K) values also need to be corrected for the 10 current CPI 2020 year and PPP; however, standard/country specific or fixed WTP values only

11 need PPP correction. The variance is calculated as follows:

$$
\operatorname{Var}_{P P P_{2020}}=\operatorname{Var}_{\text {Euros }_{2012}} x\left(\frac{C P I_{\text {Euros }_{2020}}}{C P I_{\text {Euros }_{2012}}} \times \frac{1}{P P P_{2020}}\right)^{2}-----(1)
$$

\section{Estimation of INB and its variance}

After currency conversions for cost and K, the INB can be further estimated as follows:

$$
\begin{aligned}
& I N B=K x \Delta E-\Delta C---------------(2) \text { or } \\
& I N B=\Delta E(K-I C E R)-------------(3)
\end{aligned}
$$

where $\mathrm{K}$ is the WTP, and $\Delta \mathrm{C}$ and $\Delta \mathrm{E}$ are incremental cost and incremental effectiveness respectively.

A positive INB favours treatment, i.e., intervention is cost-effective, whereas a negative INB favours the comparator, i.e., intervention is not cost-effective $[4,7,8]$. 
1 Variance of INB can be estimated as follows:

2

$$
\operatorname{Var}(I N B)=K^{2} \sigma_{\Delta E}^{2}+\sigma_{\Delta C}^{2}-2 K \sigma_{\Delta E \Delta C}------(4) \text { or }
$$

$$
\operatorname{Var}(I N B)=K^{2} \sigma_{\Delta E}^{2}+\sigma_{I C E R}^{2}-----------(5)
$$

3

4 Where $\sigma_{\Delta C}^{2}, \sigma_{\Delta E}^{2}, \sigma_{\triangle E \Delta C}$ are variances of $\Delta \mathrm{C}$ and $\Delta \mathrm{E}$ and their covariance, and $\sigma_{I C E R}^{2}$ is variance 5 of ICER. However, economic studies can report many different parameters; the 5 scenarios

6 below show how to obtain INB and variance starting with different reported data [20].

7

8 Scenario-1: The primary EES ideally reports the point estimates and variances for every 9 parameter required for calculation of INB and its variance. The INB can be calculated accordingly to equations (2) to (5)

11 Scenario-2: The study reports the means and measures of dispersion (95\% CIs) of incremental costs \& outcomes, and ICER. The variance of the ICER can be calculated using the following formulas:

14

$$
\begin{aligned}
95 \% C I \text { of } \mu_{\text {ICER }} & =\hat{\mu}_{I C E R} \pm Z_{\alpha / 2} x S E \\
U L_{I C E R} & =\hat{\mu}_{I C E R}+Z_{\alpha / 2} x S E \\
S E & =\frac{U L_{I C E R}-\hat{\mu}_{I C E R}}{Z_{\alpha / 2}}------------(6) \\
\hat{\sigma}_{I C E R}^{2} & =S E^{2} \\
U L_{I C E R} & =\text { Upper limit of ICER } \\
Z_{\alpha / 2} & =\text { Standardize normal }=1.96 \\
\hat{\mu}_{I C E R} & =\text { mean ICER }
\end{aligned}
$$

16 Once we know the variance of the ICER, the variance of the INB can be estimated using 17 equation (5). 
1 Scenario-3: The study reports means and 95\% CI, SD/SE of costs/outcomes, or $\Delta \mathrm{C} / \Delta \mathrm{E}$, but does not provide the ICER or its variance. Data for $\Delta \mathrm{C}$ and $\triangle \mathrm{E}$ are then used to simulate $\Delta \mathrm{C}$ and $\Delta \mathrm{E}$ with 1000 replications using Monte Carlo methods with a gamma and normal distributions for $\Delta \mathrm{C}$ and $\Delta \mathrm{E}$, respectively. The covariance $\left(\sigma_{\Delta E \Delta C}\right)$ between $\Delta \mathrm{C}$ and $\Delta \mathrm{E}$ as well as $\hat{\sigma}_{\Delta C}^{2} \& \hat{\sigma}_{\Delta E}^{2}$ can be then estimated. If the $95 \% \mathrm{CI}$ is provided, this is converted to SE using equation (6) above, and used to simulate data. The INB and its variance can be further calculated using equations (2) and (5).

Scenario-4: The study does not report any dispersion, but does provide the CE plane graphs, a scatter plot of $\Delta \mathrm{C}$ on $\mathrm{Y}$-axis and $\Delta \mathrm{E}$ on $\mathrm{X}$-axis, in which individual values of $\Delta \mathrm{C}$ and $\Delta \mathrm{E}$ data can be manually extracted from the CE plane using Web-Plot-Digitizer software [21]. Then, means of $\Delta \mathrm{C}, \Delta \mathrm{E}$, and their variances and co-variances can be estimated accordingly. Finally, the INB and its variance can be estimated using equations (2) and (5).

Scenario-5: The study reports neither any dispersion nor the CE-plane graph, but only provides the means (or point estimates) of costs, outcomes, and ICER. In such situations, the measures of dispersions can be borrowed from another similar study if they fulfil the following criteria:

- They are in the same stratum of country income,

- Their ICERs are not much different, e.g., $\pm 50 \%$ to $75 \%$

- They are similar in intervention, comparator, time period, country region

- Similar model type and inputs (i.e., discounting, time horizon).

If there is more than one study that meets the criteria, the average of the variances of those studies can be used.

\section{Step 3: Pooling INB}

When pooling INBs from many studies, we strongly recommend stratifying by level of country income, model type, time horizon, and perspective in order to reduce heterogeneity. The 
1 country income should be classified as low (LIC), lower-middle (LMIC), upper-middle

2 (UMIC) and high (HIC) as per the World Bank classification ${ }^{8}$. Economic models can include

3 Markov, decision tree, discrete event simulation, or others. Study perspective should include

4 societal, third-party payer, and patient perspectives. Time horizon should be lifetime and non-

5 lifetime (e.g., 5-, 10-years, etc.).

6 The INB can be pooled across studies using a fixed-effect or a random-effect model depending

7 on the degree of heterogeneity $[4,12,13,20]$.

8

9

10

11

12

A) Fixed-effects model

$$
\begin{aligned}
& \mathrm{INB}_{\mathrm{p}}=\frac{\sum_{\mathrm{i}=1}^{\mathrm{S}} \mathrm{w}_{\mathrm{i}} \cdot \mathrm{INB} \mathrm{I}_{\mathrm{i}}}{\sum_{\mathrm{i}=1}^{\mathrm{S}} \mathrm{w}_{\mathrm{i}}} \\
& w_{i}=\frac{1}{\operatorname{Var}\left(I N B_{i}\right)}-\cdots
\end{aligned}
$$

B) Random-effects model

$$
\begin{aligned}
& \mathrm{INB}_{\mathrm{p}}=\frac{\sum_{\mathrm{i}=1}^{\mathrm{S}} w_{i}^{*} \cdot \mathrm{INB}_{\mathrm{i}}}{\sum_{\mathrm{i}=1}^{\mathrm{S}} w_{i}^{*}} \\
& w_{i}^{*}=\frac{1}{\operatorname{Var}\left(I N B_{i}\right)+\tau^{2}} \\
& \tau^{2}=\frac{Q-(S-1)}{\sum w_{i}-\frac{\sum w_{i}^{2}}{\sum w_{i}}}
\end{aligned}
$$

Similar to MA in other areas, heterogeneity needs to be assessed before pooling INB.

Heterogeneity can be visualized by inspection of the forest plot, and quantitated by using the Cochrane-Q test and the $\mathrm{I}^{2}$ statistic $[4,12,13,20]$.

$$
\begin{aligned}
& \mathrm{Q}=\sum_{\mathrm{i}=1}^{\mathrm{S}} \mathrm{W}_{\mathrm{i}}\left(\mathrm{INB}_{\mathrm{i}}-\mathrm{INB}_{p}\right)^{2} \\
& \mathrm{I}^{2}=100 \% \mathrm{x} \frac{\mathrm{Q}-(\mathrm{S}-1)}{\mathrm{Q}}-\text {--.---.-. }
\end{aligned}
$$


1 If heterogeneity is present, i.e., the $\mathrm{I}^{2} \geq 25 \%$ or $\mathrm{p}$-value of $\mathrm{Q}$ test is less than 0.1 , the INBs can

2 be pooled using a random-effects model, otherwise a fixed-effect model can be applied [4, 12, $13,20]$. Exploring source/s of heterogeneity is strongly recommended. This can be done using

4 a meta-regression to fit each potential source (e.g., time horizon, percent discount rate, 5 threshold values, source of effectiveness measure, etc.) one-by-one. If that potential factor 6 explains some proportion of the heterogeneity, including it in the meta-regression model should 7 reduce the $\mathrm{I}^{2}$ accordingly. There are no established criteria how much $\mathrm{I}^{2}$ should be decreased by to consider that factor as a significant source of heterogeneity. In our experience, if the $\mathrm{I}^{2}$ is reduced by about $50 \%$ or more from the baseline model (i.e., the model without any factor), such factor/s may be source/s of heterogeneity. A post-hoc subgroup analysis by that factor should be performed accordingly. In addition, sensitivity analyses excluding a few studies with very different characteristics compared to the rest can be used to see if heterogeneity of INBs can be reduced.

Similar to general MA, publication bias should be assessed using a funnel plot and Egger's test.

A funnel plot graphs INB estimates on the $\mathrm{x}$-axis against their precision on $\mathrm{y}$-axis. If all studies are estimating the same true INB, their INBs should be randomly scattered around the true value and form a funnel shape. Egger's test formally tests if the funnel is symmetrical; if this is significant, it usually indicates that there is heterogeneity or missing studies (publication bias) or both. A contour-enhanced funnel plot is further recommended [22]. This plot will contour area of the funnel into non-significant $(\mathrm{P}$-value $>0.05-<0.1)$ and significant areas $(\mathrm{P}-$ value $<0.01$ and $<0.05$ ), which will help to differentiate the cause of the asymmetry. For instance, if missing studies fall into the non-significant area, asymmetry might be due to missing studies or publication bias. Conversely, if missing studies are in significant areas, heterogeneity is more likely to be the explanation. 
2 Example: We used data from a MA of CUA of glucagon-like peptide 1 agonists (GLP1) for

\section{---Table 1 here---}

In terms of preparing the data for pooling, 7, 1, and 2 studies provided data matching scenarios 3, 4, and 5, respectively (Table 1). Data for mean cost, QALY, and their incremental values are described in Table 2. Costs and WTP thresholds from each study were converted to \$US currency using PPP adjusted for the year 2019 using formula (1).

For the 7 studies matching scenario 3 (where mean $\mathrm{C}$ and $\mathrm{E}$ data along with SDs were reported), the Monte-Carlo method was used to simulate 1000 replicated-data based on gamma and normal distributions for cost and QALY data, respectively. Then, $\Delta \mathrm{C}$ and $\Delta \mathrm{E}$ along with variance and co-variance $\left(\sigma_{\triangle E \Delta C}\right)$ were calculated. The INB and variance was then calculated following formulas (2) and (4).

The study matching scenario 4 provided CE-plane graphs. Data for $\Delta \mathrm{C}$ and $\Delta \mathrm{E}$ were directly extracted from the CE plane using Web-Plot-Digitizer [21]. Then, variance and covariance ( $\sigma_{\triangle E \Delta C}$ ) were calculated leading to estimation of the INB and its variance using formulas (2) and (4). 
1 For the 2 studies [23, 24] matching scenario 5, the INB variance was adopted from other studies

2 following the steps outlined above. Of the 10 included studies, 2 other studies [25, 26] were

3 also conducted in the USA. For the selection of variance values for the Guillermin et al

4 study[24], the study period, time-horizon, study perspective, ICER values, drug comparison

5 (Sitagliptin) were most similar to Lee et al [26] (Table 1 and Table 2) and hence the INB

6 variance value of the latter was used to estimate the former. The values for Lee et al also

7 matched the second study [23] most closely.

8

9

--- Table 2 here----

INB data along with variances are shown in Table 3. These were then pooled across studies using a fixed-effects (inverse variance) model yielding a pooled INB (95\% CI) of US\$ 4012.21 $(-571.43,8595.85)$ with $\mathrm{I}^{2}$ of $0 \%$, see Figure 1a. In the presence of heterogeneity, as indicated by $\mathrm{I}^{2} \geq 25 \%$ or Cochrane-Q $\mathrm{p}<0.1$, a random-effects model (DerSimonian and Laird model) could be used [27]. The pooled INB value is positive but its 95\% CI covers 0, i.e., GLP1 agonists might be cost-effective as compared to DPP4 inhibitors but the results did not reach statistical significance.

---Table 3 here---

The robustness of the pooled INB as well as heterogeneity can be assessed using various sensitivity and subgroup analyses. Sensitivity analyses omitting the study that used a societal perspective [28] and the study that did not use discounting [25] yielded a pooled INB of US\$ 4,032.07 (US\$-554.48, US\$8,618.61) and US\$4,068.19 (US\$-650.66, US\$8787.04), respectively.

The WTP threshold used for these comparisons ranged from US\$29,382 to US\$ 58,024 with a median of US $\$ 49,325$. Subgroup analyses by median WTP threshold ( $<$ vs $\geq$ US $\$ 49,325$ ), time horizon, and source of effectiveness measure were performed (see Table 4), indicating 
1 GLP1s were not significantly cost-effective compared with DPP4i in any subgroup. In all these

2 sensitivity and subgroup analyses, the results were similar to that of the overall pooled INB

3 indicating that the results are robust.

--- Table 4 here---

As in general MA, publication bias was assessed using a funnel plot and Egger's test. There was no evidence of publication bias, seen either by asymmetry on the funnel plot (Figure 1b) or an Egger's test (coefficient $=0.32, \mathrm{SE}=0.73, \mathrm{p}=0.672$ ).

\section{Discussion}

We have developed MA methods for EESs focusing on data harmonisation; methodological issues include currency, time, discount, perspective, time horizon, and model used. INB and its variance are estimated based on 5 scenarios. MA is then applied to pool INBs across studies providing a summary estimated CE of treatment relative to control. This evidence should be useful for policy makers in making decisions regarding reimbursement of treatments to population in countries where resources are limited.

Despite the existence of several guidelines for reporting EESs, studies still vary in how they report the results. This data harmonisation process reported here under the 5 scenarios can help prepare data to calculate and pool INB values. The different monetary units and year can all be converted to a common standard currency.

We used INB instead of ICER as the economic effect measure because of limitations of the ICER in ambiguity of interpretation for negative ICER as mentioned above $[4,6]$. On the other hand, positive INBs indicate cost-effectiveness, while negative INBs shows non-costeffectiveness. This information will be required by policy makers $[7,8]$ in making decision from both resource-rich and resource-poor countries. 
1 A few challenges should be highlighted when applying a MA in EESs. First, EEs are

2 heterogeneous, which can be caused by model type, population, country income, GDP,

3 perspective, time horizon, and discount rate. We applied the CPI and PPP to harmonise

4 different economic backgrounds as well as the time-lag across the studies [29, 30]. However,

5 it should be noted that using PPP may have some limitations as for the estimation method of

6 price indices, which are calculated from individual prices of only selected commodities rather

7 than all commodities in each country [31]. Considering not only country income, but also

8 model type, time horizon, and perspective in stratified analyses may also reduce heterogeneity,

9 if there are sufficient data for stratifying. Furthermore, sub-group and/or sensitivity analyses

10 should be performed to identify specific types of studies/country income where treatments

11 show cost-effectiveness. Economic factors should be considered for subgroups, including WTP, discount rate, type of EES (e.g., within-trial EES versus model-based EES), quality of EESs, type of health state, and percent herd immunity for vaccine, etc.). Different subgroups of these factors may result in different cost-effectiveness findings within HICs and UMICs. Second, the health EESs are context specific, usually conducted in individual country settings. However, not all countries have EESs that fit their context because conducting well-designed EESs is very resource intensive and requires specialised expertise in economic evaluation. Therefore, there will be an even greater need for some systematic synthesis of evidence where resources are limited. Evidence from a MA will be useful if it is performed with a sensitivity to country contexts (e.g., country income, type of model, life-time, perspective, etc.).

In conclusion, we have demonstrated a tutorial of MA in EESs by applying the general methods of MA, additional with specific issues for EESs. The step-by-step approach of data harmonization is demonstrated for facilitating the process of MA. Although, evidence of CE is context specific for each country, conducting such specific individual study is challenging as 
1 similar to $\mathrm{CE}$ studies due to various practical limitations (e.g., trained man-power, time,

2 resources, etc.). Thus, the MA of EESs should be encouraged, evidence synthesis would be of

3 immense values for policy decision making process as well as aid in comparability of such

4 evidences across countries with similar contexts.

5

6

7

8

9

10

11

12

13

14

15

16

17

18

19

20

21

22

23

24

25

Page | 15 
2

3

4

5

6

7

8

9

10

11

- $\quad \Delta \mathbf{C}$ - incremental cost

- $\Delta \mathbf{E}$ - incremental effectiveness

- BMI - body mass index

- CE- cost effectiveness

- CE-plane - cost effectiveness plane

- CI - confidence interval

- CPI - consumer price index

- CEA - cost effectiveness analysis

- COMER - COMparative Efficiency Research

- CUA - cost utility study

- DALY - disability adjusted life year

- DPP4i - dipeptidyl peptidase 4 inhibitors

- EES- economic evaluation studies

- GDP - gross domestic product

- GLP1i - glucagon-like peptide-1

- HIC - high income countries

- ICER - incremental cost effectiveness ratio

- INB - incremental net benefit

- LIC - low income countries

- MA- meta analysis

- PPP- purchasing power parity

- PRISMA-P - Preferred Reporting Items for Systematic Reviews and Meta-Analyses protocol

- PSA - probabilistic sensitivity analysis

- SD - standard deviation

- $\mathbf{S E}$ - standard error

- QALY - quality adjusted life years

- UMIC - upper middle-income countries

- US - united states

- WHO - world health organisation

- $\mathbf{W T P}$ - willingness to pay 
- Ethics approval and consent to participate: Not Applicable

- Consent for publication: Not Applicable

- Availability of data and material: All the relevant data is included in the manuscript

- Competing interests: None

- Funding: This work is a part of training in Health Technology Assessment's master's degree, in which scholarship is provided by Mahidol University and the International Decision Support Initiative (iDSI). This work was produced as part of the International Decision Support Initiative ( www. idsihealth. org), which supports countries to get the best value for money from health spending. iDSI receives funding support from the Bill and Melinda Gates Foundation, the UK Department for International Development, and the Rockefeller Foundation.

- Authors' contributions:

1) Conceived and designed the experiments: BSB, AT

2) Performed the experiments: BSB, AT

3) Analyzed and interpreted the data: BSB, UC, AT

4) Contributed reagents, materials, analysis tools or data: BSB, UC, NC, JA, AT

5) Wrote the paper: BSB, UC, NC, JA, AT

- Acknowledgements: BSB acknowledged the financial support through a fellowship by the department of health research, government of India under Human Resource Development program, Indian Council of Medical Research. This article is an important part of the training of BSB, who is a master's student in health technology assessment at Mahidol University, Bangkok, Thailand. 
References:

1. Drummond MF, Sculpher MJ, Torrance GW, O'Brien BJ, Stoddart GL: Methods for the Economic Evaluation of Health Care Programmes, 3 edition edn. Oxford: Oxford University Press; 2005.

2. Torrance GW: Measurement of health state utilities for economic appraisal. $J$ Health Econ 1986, 5(1):1-30.

3. Wisloff $T$, Hagen G, Hamidi V, Movik E, Klemp M, Olsen JA: Estimating QALY gains in applied studies: a review of cost-utility analyses published in 2010. Pharmacoeconomics 2014, 32(4):367-375.

4. Crespo C, Monleon A, Diaz W, Rios M: Comparative efficiency research (COMER): metaanalysis of cost-effectiveness studies. BMC Med Res Methodol 2014, 14:139.

5. O'Mahony JF: The Limitations of Icers In Screening Interventions and The Relative Net Benefit Alternative. Value Health 2015, 18(7):A705.

6. Hoch JS, Dewa CS: Advantages of the net benefit regression framework for economic evaluations of interventions in the workplace: a case study of the cost-effectiveness of a collaborative mental health care program for people receiving short-term disability benefits for psychiatric disorders. J Occup Environ Med 2014, 56(4):441-445.

7. Willan AR: Incremental net benefit in the analysis of economic data from clinical trials, with application to the CADET-Hp trial. Eur J Gastroenterol Hepatol 2004, 16(6):543-549.

8. Willan AR, Lin DY: Incremental net benefit in randomized clinical trials. Stat Med 2001, 20(11):1563-1574.

9. Higgins JPT TJ, Chandler J, Cumpston M, Li T, Page MJ, Welch VA (editors): Cochrane Handbook for Systematic Reviews of Interventions version 6.1 (updated September 2020); 2020.

10. Normand SL: Meta-analysis: formulating, evaluating, combining, and reporting. Stat Med 1999, 18(3):321-359.

11. Shamseer L, Moher D, Clarke M, Ghersi D, Liberati A, Petticrew M, Shekelle P, Stewart LA, Group P-P: Preferred reporting items for systematic review and meta-analysis protocols (PRISMA-P) 2015: elaboration and explanation. BMJ 2015, 350:g7647.

12. Bagepally BS, Chaikledkaew U, Gurav YK, Anothaisintawee T, Youngkong S, Chaiyakunapruk N, McEvoy M, Attia J, Thakkinstian A: Glucagon-like peptide 1 agonists for treatment of patients with type 2 diabetes who fail metformin monotherapy: systematic review and metaanalysis of economic evaluation studies. BMJ Open Diabetes Res Care 2020, 8(1).

13. Bagepally BS, Gurav YK, Anothaisintawee T, Youngkong S, Chaikledkaew U, Thakkinstian A: Cost Utility of Sodium-Glucose Cotransporter 2 Inhibitors in the Treatment of Metformin Monotherapy Failed Type 2 Diabetes Patients: A Systematic Review and Meta-Analysis. Value Health 2019, 22(12):1458-1469.

14. Haider S, Chaikledkaew U, Thavorncharoensap M, Youngkong S, Islam MA, Thakkinstian A: Systematic Review and Meta-Analysis of Cost-effectiveness of Rotavirus Vaccine in LowIncome and Lower-Middle-Income Countries. Open Forum Infect Dis 2019, 6(4):ofz117.

15. Meta-analysis of economic evaluations. In: WEEKLY EPIDEMIOLOGICAL RECORD. Volume No 17,, edn. Edited by (IVIR-AC) IaVIRAC. Meeting of the Immunization and Vaccinerelated Implementation Research Advisory Committee (IVIR-AC), March 2021: World Health Organisation; 2021: 138-140.

16. Woods B, Revill, P., Sculpher, M., Claxton, C.: Country-level Cost-Effectiveness Thresholds: Initial Estimates and the Need for Further Research. CHE Research Paper 2016:109.

17. Robinson LA, Hammitt JK, Chang AY, Resch S: Understanding and improving the one and three times GDP per capita cost-effectiveness thresholds. Health Policy and Planning 2016, 32(1):141-145. 
18. Marseille E, Larson B, Kazi DS, Kahn JG, Rosen S: Thresholds for the cost-effectiveness of interventions: alternative approaches. Bull World Health Organ 2015, 93(2):118-124.

19. IMF data [https://www.imf.org/external/pubs/ft/weo/2018/01/weodata/index.aspx]

20. Haider S, Chaikledkaew U, Thavorncharoensap M, Youngkong S, Islam MA, Thakkinstian A: Systematic Review and Meta-Analysis of Cost-effectiveness of Rotavirus Vaccine in LowIncome and Lower-Middle-Income Countries. Open Forum Infectious Diseases 2019, 6(4).

21. WebPlotDigitizer - Extract data from plots, images, and maps [https://automeris.io/WebPlotDigitizer/download.html]

22. Peters JL, Sutton AJ, Jones DR, Abrams KR, Rushton L: Contour-enhanced meta-analysis funnel plots help distinguish publication bias from other causes of asymmetry. $J$ Clin Epidemiol 2008, 61(10):991-996.

23. Sinha $A$, Rajan $M$, Hoerger $T$, Pogach $L$ : Costs and consequences associated with newer medications for glycemic control in type 2 diabetes. Diabetes Care 2010, 33(4):695-700.

24. Guillermin AL, Lloyd A, Best JH, DeYoung MB, Samyshkin Y, Gaebler JA: Long-term costconsequence analysis of exenatide once weekly vs sitagliptin or pioglitazone for the treatment of type 2 diabetes patients in the United States. J Med Econ 2012, 15(4):654-663.

25. Bruhn D, Martin AA, Tavares R, Hunt B, Pollock RF: Cost-utility of albiglutide versus insulin lispro, insulin glargine, and sitagliptin for the treatment of type 2 diabetes in the US. J Med Econ 2016, 19(7):672-683.

26. Lee WC, Samyshkin Y, Langer J, Palmer JL: Long-term clinical and economic outcomes associated with liraglutide versus sitagliptin therapy when added to metformin in the treatment of type 2 diabetes: a CORE Diabetes Model analysis. J Med Econ 2012, 15 Suppl 2:28-37.

27. DerSimonian R, Laird N: Meta-analysis in clinical trials. Control Clin Trials 1986, 7(3):177-188.

28. Steen Carlsson K, Persson U: Cost-effectiveness of add-on treatments to metformin in a Swedish setting: liraglutide vs sulphonylurea or sitagplitin. J Med Econ 2014, 17(9):658-669.

29. Problems and Extensions of PPP [http://internationalecon.com/Finance/Fch30/F30-4.php]

30. Burdick C, Fisher L: Social Security cost-of-living adjustments and the Consumer Price Index. Soc Secur Bull 2007, 67(3):73-88.

31. Pigou AC: The Foreign Exchanges. The Quarterly Journal of Economics 1922, 37(1):52-74.

Figure legends

Figure 1: a) Forrest plot of pooling INBs of GLP1 vs DPP4i; b) Funnel plot of pooling INB of GLP1 vs DPP4i. 
Figures

a) Forest Plot

Study

Sinha A (2010)

Davies MJ (2012)

Guillermin AL (2012)

Lee WC (2012)

Mezquita Raya P (2013)

Steen Carlsson K (2014)

Perez A (2015)

Bruhn D (2016)

Roussel R (2016)

Barnett AH (2018)

Overall

Heterogeneity: $I^{2}=0.00 \%, \mathrm{H}^{2}=1.00$

Test of $\theta_{1}=\theta_{i}: Q(9)=5.69, p=0.77$

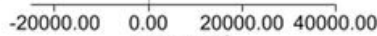

INB in US\$ b) Funnel Plot

INB in US\$ with $95 \% \mathrm{Cl}$

\begin{tabular}{r}
$\begin{array}{c}\text { Weight } \\
(\%)\end{array}$ \\
\hline 7.21 \\
17.89 \\
7.21 \\
7.21 \\
14.93 \\
0.13 \\
7.61 \\
5.65 \\
9.87 \\
22.30 \\
\end{tabular}

Fixed-effects inverse-variance mode

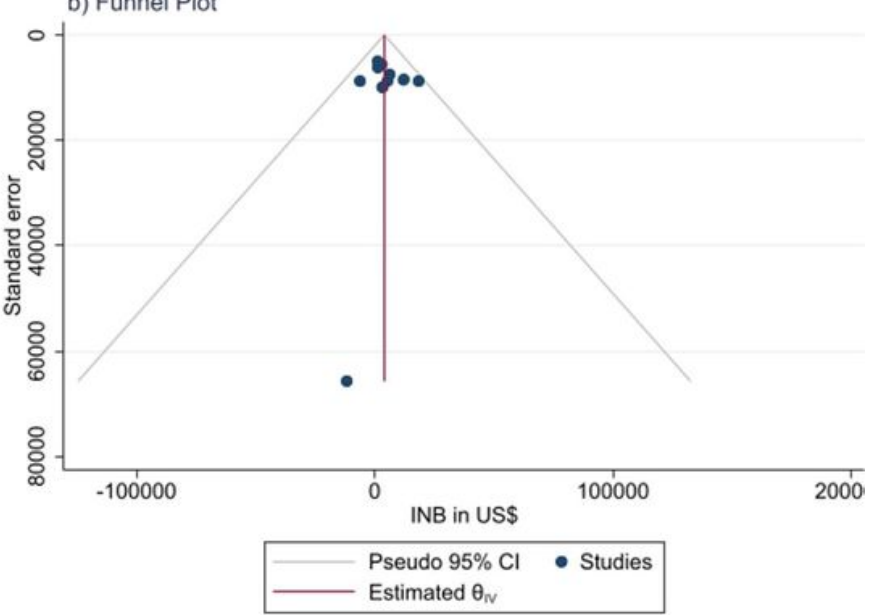

Figure 1

a) Forrest plot of pooling INBs of GLP1 vs DPP4i; b) Funnel plot of pooling INB of GLP1 vs DPP4i. 